\title{
Neoclassical Pitch-Angle Scattering of Runaway Electrons
}

\author{
Jian Liu, ${ }^{1,2}$ Yulei Wang, ${ }^{1,2}$ and Hong Qin ${ }^{1,3, * *}$ \\ ${ }^{1}$ School of Nuclear Science and Technology and Department of Modern Physics, \\ University of Science and Technology of China, Hefei, Anhui 230026, China \\ ${ }^{2}$ Key Laboratory of Geospace Environment, CAS, Hefei, Anhui 230026, China \\ ${ }^{3}$ Plasma Physics Laboratory, Princeton University, Princeton, NJ 08543
}

\begin{abstract}
It is discovered that the tokamak field geometry generates a pitch-angle scattering effect for runaway electrons. This neoclassical pitch-angle scattering is much stronger than the collisional scattering and invalidates the gyro-center model for runaway electrons. As a result, the energy limit of runaway electrons is found to be larger than the prediction of the gyro-center model and to depend heavily on the background magnetic field.
\end{abstract}

\footnotetext{
* Corresponding author. hongqin@ustc.edu.cn
} 
Runaway electrons in a tokamak are energetic particles accelerated by the electric field. They cannot be braked by the collisional drag [1]. A large amount of runaway electrons are produced in tokamaks during fast shutdowns, disruptions [2 15], or aggressive current drive [16]. The massive energy carried by runaway beams poses great danger to plasmafacing components $[17-20]$. Understanding the physics of runaway electrons in toroidal field configurations is thus critical. The dynamics of runaway electrons involves different timescales spanning 11 orders of magnitude, which brings difficulties to both analytical and numerical studies. Gyro-center model is often applied to tackle the multi-scale problem by averaging out the fast gyro-motion and has produced fruitful results [21 29]. According to the gyro-center model, the magnetic moment of runaway electron is an adiabatic invariant, and the parallel momentum increases due to the work by the loop electric field. There is no channel of momentum transfer from the parallel to the perpendicular direction, except for the collision with background plasmas. In general, the collisional effect is rather weak for charged particles with high velocities [25]. For a typical runaway electron, the collision time is $\tau_{c o l} \sim 0.5 \mathrm{~s}$, which is much longer than the gyro-period $T_{c e}\left(\sim 10^{-10} \mathrm{~s}\right)$ and the transit period $T_{t r}\left(\sim 10^{-8} \mathrm{~s}\right)$. When the collisional effect can be neglected, the perpendicular momentum will monotonically decrease due to the synchrotron radiation of the gyro-motion [25], and parallel momentum will monotonically increase to its maximum limit until the electric field acceleration is finally balanced by the radiation dissipation. The pitch-angle scattering due to collisions will transfer a small amount of energy from the parallel direction to the perpendicular direction. This small collisional effect keeps the runaway electrons energetic in the perpendicular direction, but does not modify the energy limit in the parallel direction by much. The gyro-center model predicts that the energy limit of runaway electrons does not depend on collisions and the magnitude of background magnetic field [21].

Contrary to the common wisdom, our analysis shows that the gyro-center model is not valid for runaway electrons. This is because they move with the speed of light in the parallel direction, the local magnetic field they see changes by a large amount during one gyroperiod. Therefore, the basic assumption for the gyro-center model, i.e., the magnetic field is approximately constant in one gyro-period, breaks down. In fact, the magnetic moment $\mu$ is no longer an adiabatic invariant. Similar non-conservation of $\mu$ has been observed in the presence of magnetic turbulence with wavelength comparable to the gyro-radius [30, $\underline{31}$ ].

In this letter, we abandon the gyro-center model and study the multi-timescale runaway 
dynamics by numerically solving the dynamical equations of runaway electrons directly in the six-dimensional phase space. Long-term simulation results confirm that the gyrocenter model is indeed invalid, see Fig.1, More than one hundred billion time steps are required in the simulation. To eliminate the coherent accumulation of numerical errors from each time step, which is usually a show-stopper for long-term simulations, we utilize the newly developed volume-preserving algorithm (VPA) for relativistic particles [32]. The VPA can guarantee the long-term accuracy by preserving the phase-space volume of the original physical system. Its long-term conservativeness and stability have been verified.

Taking the advantage of the VPA method, we discovered that there exists a new pitchangle scattering mechanism, which transfers momentum of runaway electrons between the parallel and perpendicular directions. It arises from the full orbit dynamics in the toroidal geometry of a tokamak, hence the name of neoclassical scattering. The neoclassical pitch-angle scattering process is about a million times faster than the collisional pitch-angle scattering, resulting in a rapid transfer of the parallel momentum, gained from the loop electric field, to the perpendicular direction. As an important result, the simulation study indicates a new energy limit for runaway electrons, which is higher than the result from the gyro-center model and varies with the magnitude of the background magnetic field. This unexpected neoclassical pitch-angle scattering effect for runaway electrons and its important consequence are the subjects of this letter.

First, we introduce the physical model. When focusing on the long-term dynamics of runaway electrons in a tokamak, we take into account the background magnetic field, the loop electric field, and the electromagnetic radiation. The dynamical equations of runaway electrons are

$$
\begin{aligned}
\frac{\mathrm{d} \mathbf{x}}{\mathrm{d} t} & =\mathbf{v} \\
\frac{\mathrm{d} \mathbf{p}}{\mathrm{d} t} & =-\mathrm{e}(\mathbf{E}+\mathbf{v} \times \mathbf{B})+\mathbf{F}_{R} \\
\mathbf{p} & =\gamma \mathrm{m}_{0} \mathbf{v}
\end{aligned}
$$

where $\mathbf{x}, \mathbf{v}, \mathbf{p}$ denote the position, velocity and mechanical momentum of a runaway electron, e denotes the unit charge, $\mathrm{m}_{0}$ is the rest mass of electron, $\mathbf{E}$ and $\mathbf{B}$ are the electric and magnetic field, and the Lorentz factor $\gamma$ is defined as

$$
\gamma=\sqrt{1+\frac{p^{2}}{\mathrm{~m}_{0}^{2} \mathrm{c}^{2}}}=\frac{1}{\sqrt{1-(v / \mathrm{c})^{2}}}
$$


The effective electromagnetic radiation drag force $\mathbf{F}_{R}$ is

$$
\mathbf{F}_{R}=-P_{R} \frac{\mathbf{v}}{v^{2}}
$$

where $P_{R}$ is the radiation power determined by [33]

$$
P_{R}=\frac{q_{e}^{2}}{6 \pi \epsilon_{0} \mathrm{c}} \gamma^{2}\left[\left(\frac{\mathbf{a}}{\mathrm{c}}\right)^{2}-\left(\frac{\mathbf{v}}{\mathrm{c}} \times \frac{\mathbf{a}}{\mathrm{c}}\right)^{2}\right]
$$

Here, $\epsilon_{0}$ is the permittivity in vacuum, $\mathrm{c}$ is the speed of light in vacuum, and $\mathbf{a}=\mathrm{d} \mathbf{v} / \mathrm{d} t$ denotes the acceleration.

In order to solve Eqs. (11)-(2) numerically, we have to meet the challenge brought by the multi-scale nature of the problem. Restricted by the minimal timescale, more than $10^{11}$ time-steps are required to simulate a complete runaway dynamics. Traditional algorithms, such as the 4th order Runge-Kutta method, are not qualified for this long-term simulation, because the coherent accumulation of numerical error over many time-steps leads to incorrect long-term simulation results. To overcome this difficulty, geometric algorithms which can bound the global numerical error for all time-steps should be adopted [32, 34 38]. The newly developed relativistic VPA [32] with radiation drag is utilized in the present study to guarantee the long-term numerical accuracy.

As a model, the background magnetic field and inductive electric field are set to be

$$
\begin{aligned}
& \mathbf{B}=\frac{B_{0} R_{0}}{R} \mathbf{e}_{\xi}-\frac{B_{0} \sqrt{\left(R-R_{0}\right)^{2}+z^{2}}}{q R} \mathbf{e}_{\theta}, \\
& \mathbf{E}=E_{l} \frac{R_{0}}{R} \mathbf{e}_{\xi}
\end{aligned}
$$

where $R=\sqrt{x^{2}+y^{2}}, \xi$, and $z$ are radial distance, azimuth, and height of the cylindrical coordinate system respectively, $\mathbf{e}_{\xi}$ and $\mathbf{e}_{\theta}$ are the unit vectors along toroidal and poloidal directions, and $q$ denotes safety factor. Without loss of generality, we use the parameters of EAST [39]. The major radius is $R_{0}=1.7 \mathrm{~m}$, the safety factor is $q=2$, the central magnetic field is $B_{0}=3 \mathrm{~T}$, and the loop electric field is $E_{l}=0.2 \mathrm{~V} / \mathrm{m}$. The initial parallel and perpendicular momentum of a typical runaway electron are set to be $p_{\| 0}=5 \mathrm{~m}_{0} \mathrm{c}$ and $p_{\perp 0}=1 \mathrm{~m}_{0} \mathrm{c}$, and the initial position is $R=1.8 \mathrm{~m}$ and $\xi=z=0$. The time-step of simulation is set to $\Delta t=1.9 \times 10^{-12} \mathrm{~s}$, which is about $1 \%$ of the gyro-period.

To verify that the condition for gyro-center approximation is not satisfied for runaway electrons in tokamaks, we record the change ratio of the magnetic field $\Delta_{B}(t)$ that one 


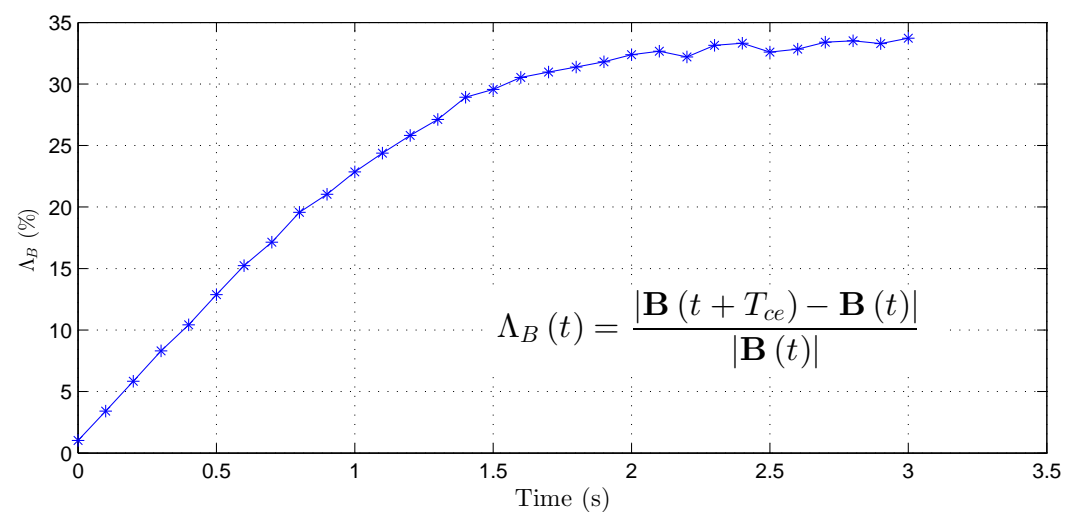

FIG. 1. The change ratio of background magnetic field $\Delta_{B}$ during one gyro-period at different time of a runaway dynamics.

runaway electron samples during one gyro-period at different time $t$, see Fig.1. The change ratio is defined as

$$
\Delta_{B}(t)=\frac{\left|\mathbf{B}\left(t+T_{c e}\right)-\mathbf{B}(t)\right|}{|\mathbf{B}(t)|},
$$

where $T_{c e}=2 \pi \gamma \mathrm{m}_{0} / e B$ is the gyro-period. For the gyro-center approximation to be valid, the variation of the magnetic field a particle samples during one gyro-period should be small. However, simulation results show that the change ratio $\Delta_{B}$ increases monotonously with the runaway energy. Its value increases to $10 \%$ after $0.4 \mathrm{~s}$ and exceeds $30 \%$ after $1.7 \mathrm{~s}$, which can no longer be taken as a small value. This is mainly because the velocity of runaway electron approaches the speed of light, and the gyro-period $T_{c e}$ is proportional to the Lorentz factor $\gamma$. The runaway electron travels a long distance along the toroidal direction during each gyro-period, which leads to a large value of $\Delta_{B}$.

The momentum evolution of runaway electrons is plotted in Fig. 2. The increase of the parallel momentum is similar to the results from the gyro-center model [23, 25], but the evolution of the perpendicular momentum is very different. The perpendicular momentum grows with rapid oscillations, even in the absence of collisions, until approaching a maximum after about $2.5 \mathrm{~s}$. Because the parallel momentum is relatively large, its oscillation is less prominent. The oscillation is an effective scattering process transferring the parallel momentum to the perpendicular direction and altering the pitch-angle. As explained later, this effective pitch-angle scattering roots in the geometric configuration of the field. It is thus a neoclassical pitch-angle scattering effect. The evolution of the perpendicular momentum 

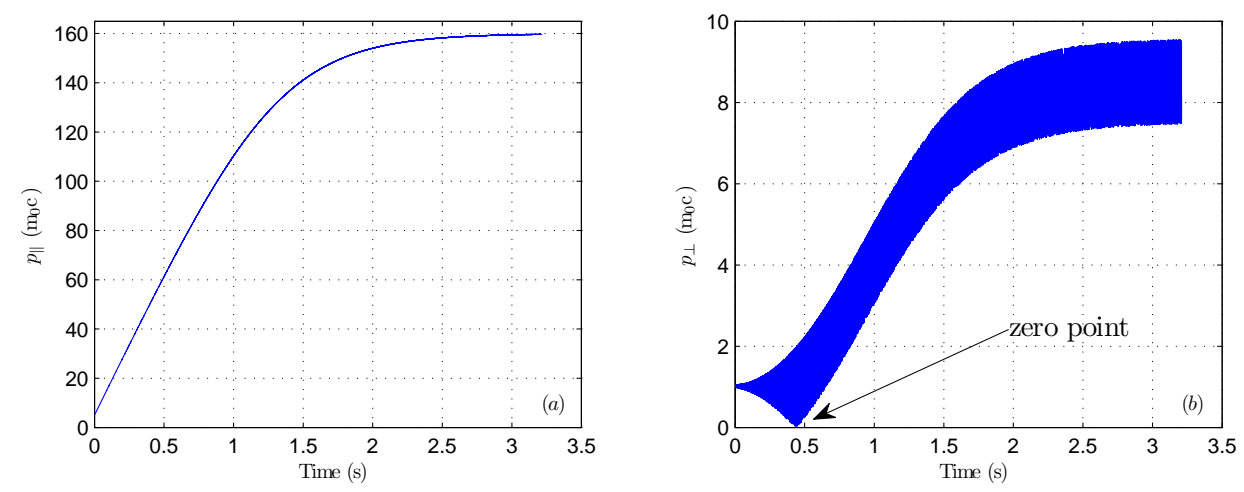

FIG. 2. The evolution of (a) parallel momentum and (b) perpendicular momentum of a runaway electron. The initial position of the electron is at $R=1.8 \mathrm{~m}, \xi=z=0$, and the initial momentum is $p_{\|}=5 \mathrm{~m}_{0} \mathrm{c}$ and $p_{\perp}=\mathrm{m}_{0} \mathrm{c}$. The center magnetic field is $B_{0}=3 \mathrm{~T}$ and the loop electric field is $E_{l}=0.2 \mathrm{~V} / \mathrm{m}$.

exhibits four stages: (a) a rapid oscillation is developed initially; (b) then its absolute value reaches the zero point; (c) it grows quickly after passing the zero point, and (d) it saturates. At the saturation, though the pitch-angle still varies quickly due to the neoclassical scattering, the average of the perpendicular momentum does not change. The timescale of neoclassical pitch-angle scattering is $5 \times 10^{-7} \mathrm{~s}$, which is about $10^{6}$ times faster than the collisional scattering, and much more momentum can be transferred to the perpendicular direction through the neoclassical scattering than the collisional effect as calculated in Ref [25].

The seemingly complex evolution curves of the perpendicular momentum are dominated by the neoclassical scattering, which can be analyzed by looking at the variation of the momentum vector. We choose the parallel moment $p_{\|}$, the z-component of the perpendicular momentum $p_{\perp z}$, and the projection of perpendicular moment in the $x-y$ plane $p_{\perp x y}$ as the three coordinates for the moment. They satisfy $p_{\perp x y}^{2}+p_{\perp z}^{2}+p_{\|}^{2}=p^{2}$. In Fig. 3, snapshots of the momentum vector within one gyro-period at different moments are plotted in the momentum space. During each gyro-period, the tip of the momentum vector moves approximately along a circular orbit. The minimal and maximal value of $p_{\perp z}$ are marked within each gyro-period. The circular orbit is first elongated while the center of circle shifts to $p_{\perp z}$ direction in the perpendicular plane. The elongation of the orbit corresponds to the growth of the oscillation amplitude. At $t=0.432 \mathrm{~s}$, the orbit touches the $p_{\perp z}=0$ plane, and 

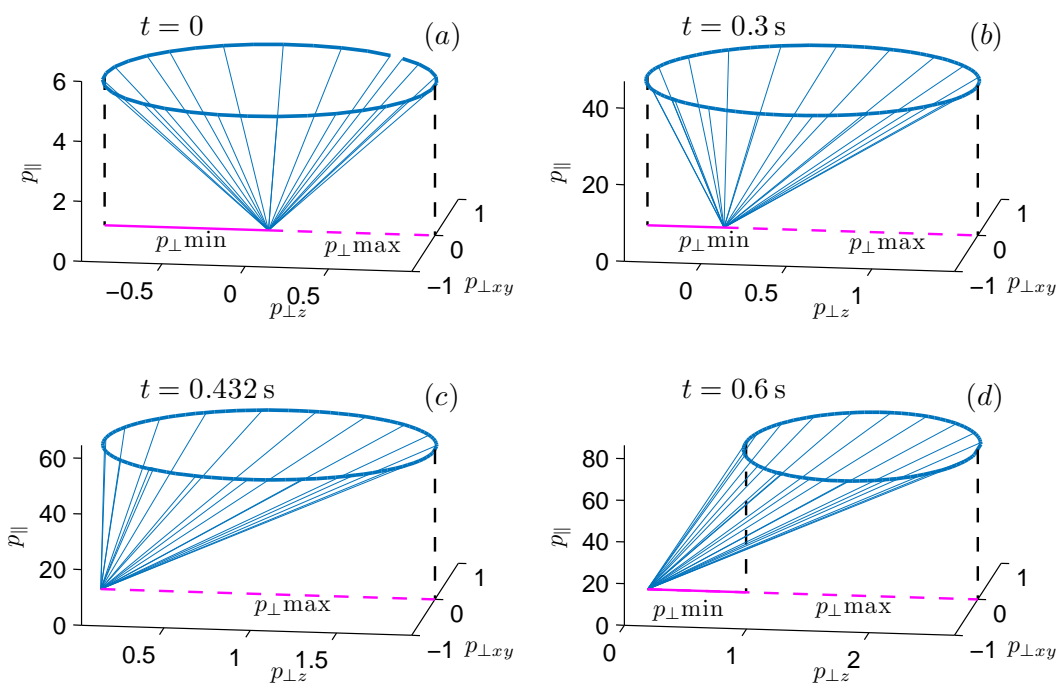

FIG. 3. Motion of the momentum vector during one gyro-period at different time. The maximal and minimal perpendicular momenta, $p_{\perp \max }$ and $p_{\perp \text { min }}$, during each gyro-period are marked by dashed line.
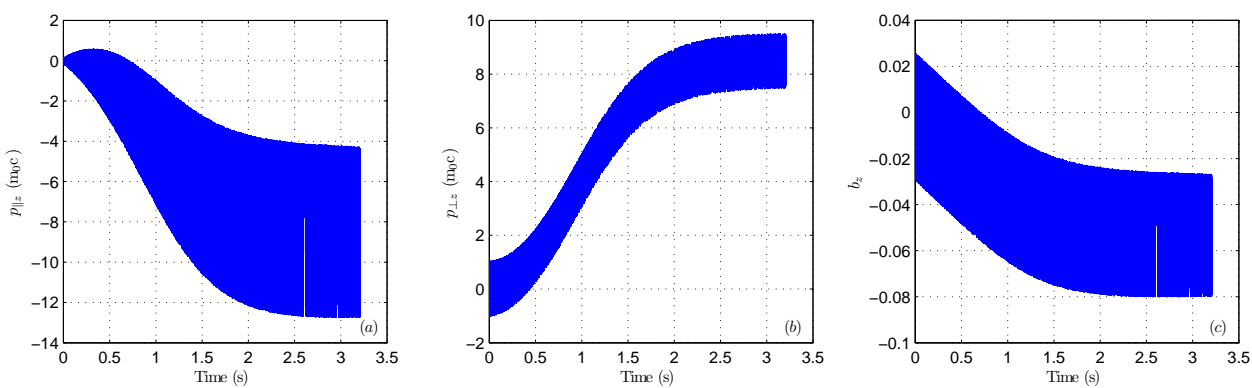

FIG. 4. Evolution of (a) z-component of parallel momentum $p_{\| z}$, (b) z-component of perpendicular momentum $p_{\perp z}$, and (c) z-component of unit vector along the magnetic field $b_{z}$.

the zero point of perpendicular momentum appears. Afterwards, the elongated orbit keeps shifting towards the larger $p_{\perp z}$ direction until approaching a steady state. It is evident that the variation of $p_{\perp}$ is mainly due to the $z$-component $p_{\perp z}$.

Figure 4 shows the evolution of the $z$-component of the parallel momentum $p_{\| z}$, the $z$ component of the perpendicular momentum $p_{\perp z}$, and the $z$-component of the unit vector along the magnetic field $b_{z}=\mathbf{b} \cdot \mathbf{z}=\mathbf{B} \cdot \mathbf{z} / B$. We find that $p_{\perp z}$ increases, oscillates, and saturates around $9 m_{0} c$. The oscillation amplitude of $p_{\| z}$ increases with the absolute value of $p_{\|}$. Meanwhile, the evolution of $p_{\| z}$ has the same trend as $b_{z}$, which indicates 


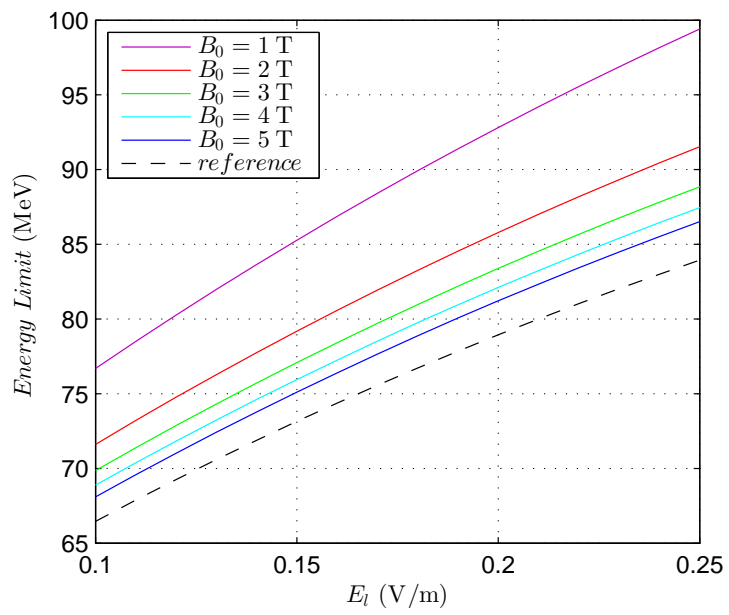

FIG. 5. Plots of energy limit versus loop electric field $E_{l}$. The black dashed curve corresponds to the energy limit curve predicted by gyro-center model [21], where collisional effect is ignorable. The solid curves correspond to energy limits with different magnetic field intensities. The loop electric field is set to be radially uniform in order to compare with the gyro-center model.

that the neoclassical scattering is closely related to the direction of the local magnetic field. Due to the neoclassical drift [25, 26], the transit orbits of runaway electrons drift outwards from the magnetic axis. As a result, runaway electrons spend more and more time in the $R>R_{0}$ region. The magnetic field runaway electrons sample then tilts more towards the negative $\mathrm{z}$ direction on average, because of the helical configuration of the magnetic field lines. Since the parallel momentum is defined to be $p_{\|}=\mathbf{p} \cdot \mathbf{b}$, the change of $\mathbf{b}$ results in a change of $p_{\|}$. In the $z$-direction, the time average of momentum vanishes approximately, i.e., $\left\langle p_{z}\right\rangle=\left\langle p_{\| z}\right\rangle+\left\langle p_{\perp z}\right\rangle=0$. Therefore, decrease of $\left\langle p_{\| z}\right\rangle$ corresponds to an increase of $\left\langle p_{\perp z}\right\rangle$.

The energy limit is reached at the saturation. The variation of the energy limit against the loop electric field with different magnetic field intensities are plotted in Fig.5, which clearly shows that the energy limit depends on the intensity of the background magnetic field. This is different from the conclusion by Martín-Solís et al. using the gyro-center model [21]. For the case of $B_{0}=1 \mathrm{~T}$, our energy limit is about $20 \%$ higher, because more energy is transferred to the perpendicular direction through the neoclassical pitch-angle scattering. If the magnetic field is extremely strong, the gyro-center approximation model will be valid, and our energy limit curve will recover the gyro-center result. 
In summary, long-term simulations using the newly developed volume preserving algorithm revealed that the full orbit dynamics of a runaway electron in the tokamak field geometry generates a pitch-angle scattering effect for runaway electrons. In a typical tokamak, this neoclassical pitch-angle scattering is about 1 million times stronger than the collisional scattering and invalidates the gyro-center model for runaway electrons. As a consequence, the energy limit of runaway electrons is found to be larger than the prediction by a gyrocenter model. In addition, the theoretical model developed in the present study shows that the runaway energy limit also depend heavily on the background magnetic field.

\section{ACKNOWLEDGMENTS}

This research is supported by National Magnetic Confinement Fusion Energy Research Project (2015GB111003, 2014GB124005), National Natural Science Foundation of China (NSFC-11575185, 11575186, 11305171), JSPS-NRF-NSFC A3 Foresight Program (NSFC11261140328), the CAS Program for Interdisciplinary Collaboration Team, and the GeoAlgorithmic Plasma Simulator (GAPS) Project.

[1] H. Dreicer, Phys. Rev. 115, 238 (1959).

[2] R. Yoshino, T. Kondoh, Y. Neyatani, et al., Plasma Phys. Control. Fusion 39, 313 (1997).

[3] R. Jaspers, N. L. Cardozo, F. Schuller, et al., Nucl. Fusion 36, 367 (1996).

[4] P. Helander, L.-G. Eriksson, and F. Andersson, Phys. Plasmas 7, 4106 (2000).

[5] P. Helander, L. Eriksson, and F. Andersson, Plasma Phys. Contr. Fusion 44, B247 (2002).

[6] T. Fülöp, H. Smith, and G. Pokol, Phys. Plasmas 16, 022502 (2009).

[7] R. Gill, B. Alper, A. Edwards, et al., Nucl. Fusion 40, 163 (2000).

[8] R. Jaspers, K. Finken, G. Mank, et al., Nucl. Fusion 33, 1775 (1993).

[9] R. Nygren, T. Lutz, D. Walsh, et al., J. Nucl. Mater. 241, 522 (1997).

[10] P. Parks, M. Rosenbluth, and S. Putvinski, Phys. Plasmas 6, 2523 (1999).

[11] M. Rosenbluth and S. Putvinski, Nucl. Fusion 37, 1355 (1997).

[12] R. Yoshino and S. Tokuda, Nucl. Fusion 40, 1293 (2000).

[13] H. Tamai, R. Yoshino, S. Tokuda, et al., Nucl. Fusion 42, 290 (2002). 
[14] M. Lehnen, S. Bozhenkov, S. Abdullaev, et al., Phys. Rev. Lett. 100, 255003 (2008).

[15] K. Finken, S. Abdullaev, M. Jakubowski, et al., Nucl. Fusion 47, 91 (2007).

[16] N. J. Fisch, Rev. Mod. Phys. 59, 175 (1987).

[17] H.-W. Bartels, Fusion Eng. Des. 23, 323 (1994).

[18] T. Kawamura, H. Obayashi, and A. Miyahara, Fusion Eng. Des. 9, 39 (1989).

[19] H. Bolt, A. Miyahara, M. Miyake, et al., J. Nucl. Mater. 151, 48 (1987).

[20] R. Jaspers, N. L. Cardozo, A. Donne, et al., Rev. Sci. Instrum. 72, 466 (2001).

[21] J. Martín-Solís, J. Alvarez, R. Sánchez, et al., Phys. Plasmas 5, 2370 (1998).

[22] J. Martín-Solís, B. Esposito, R. Sánchez, et al., Phys. Plasmas 6, 238 (1999).

[23] J. Liu, H. Qin, N. J. Fisch, et al., Phys. Plasmas 21, 064503 (2014).

[24] M. Bakhtiari, G. Kramer, M. Takechi, et al., Phys. Plasmas 12, 102503 (2005).

[25] X. Guan, H. Qin, and N. J. Fisch, Phys. Plasmas 17, 092502 (2010).

[26] H. Qin, X. Guan, and N. J. Fisch, Report No. PPPL-4639, Princeton Plasma Physics Laboratory (PPPL), Princeton, NJ (United States) (2011).

[27] G. Papp, M. Drevlak, T. Fülöp, et al., Nucl. Fusion 51, 043004 (2011).

[28] J. Rax, N. Fisch, and L. Laurent, Plasma Phys. Contr. Fusion 35, B129 (1993).

[29] F. Andersson, P. Helander, and L.-G. Eriksson, Phys. Plasmas 8, 5221 (2001).

[30] S. Dalena, A. Greco, A. Rappazzo, et al., Physical Review E 86, 016402 (2012).

[31] S. Dalena, A. Rappazzo, P. Dmitruk, et al., Astrophys. J. 783, 143 (2014).

[32] R. Zhang, J. Liu, H. Qin, et al., Phys. Plasmas 22, 044501 (2015).

[33] J. D. Jackson, Classical electrodynamics, vol. 3 (Wiley New York etc., 1962).

[34] H. Qin and X. Guan, Phys. Rev. Lett. 100, 035006 (2008).

[35] Y. He, Y. Sun, J. Liu, et al., J. Comput. Phys. 281, 135 (2015).

[36] H. Qin, S. Zhang, J. Xiao, et al., Phys. Plasmas 20, 084503 (2013).

[37] J. Xiao, J. Liu, H. Qin, et al., Phys. Plasmas 20, 102517 (2013).

[38] R. Zhang, J. Liu, Y. Tang, et al., Phys. Plasmas 21, 032504 (2014).

[39] S. Wu, Fusion Eng. Des. 82, 463 (2007). 